\title{
Isotropic-Nematic Interface and Wetting in Suspensions of Colloidal Platelets
}

\author{
D. van der Beek, ${ }^{1, *}$ H. Reich, ${ }^{2}$ P. van der Schoot, ${ }^{3}$ M. Dijkstra, ${ }^{4}$ T. Schilling, ${ }^{5}$ R. Vink, ${ }^{2}$ \\ M. Schmidt, ${ }^{2,6}$ R. van Roij, ${ }^{7}$ and H. Lekkerkerker ${ }^{1}$ \\ ${ }^{1}$ Van 't Hoff Laboratory for Physical and Colloid Chemistry, Utrecht University, Padualaan 8, 3584 CH Utrecht, The Netherlands \\ ${ }^{2}$ Institut für Theoretische Physik II, Heinrich-Heine-Universität Düsseldorf, Universitätsstrasse 1, 40225 Düsseldorf, Germany \\ ${ }^{3}$ Polymer Laboratories, Technische Universiteit Eindhoven, P. O. Box 513, 5600 MB Eindhoven, The Netherlands \\ ${ }^{4}$ Soft Condensed Matter, Utrecht University, Princetonplein 5, 3584 CC Utrecht, The Netherlands \\ ${ }^{5}$ Institut für Physik, Johannes Gutenberg-Universität, Staudinger Weg 7, 55099 Mainz, Germany \\ ${ }^{6}$ H.H. Wills Physics Laboratory, University of Bristol, Tyndall Avenue, Bristol BS8 1TL, United Kingdom \\ ${ }^{7}$ Institute for Theoretical Physics, Utrecht University, Leuvenlaan 4, 3584 CE Utrecht, The Netherlands
}

(Received 5 May 2006; published 25 August 2006)

\begin{abstract}
We study interfacial phenomena in a colloidal dispersion of sterically stabilized gibbsite platelets, exhibiting coexisting isotropic and nematic phases separated by a sharp horizontal interface. The nematic phase wets a vertical glass wall and polarized light micrographs reveal homeotropic surface anchoring both at the free isotropic-nematic interface and at the wall. On the basis of complete wetting of the wall by the nematic phase, as found in our density functional calculations and computer simulations, we analyze the balance between Frank elasticity and surface anchoring near the contact line. Because of weak surface anchoring, the director field in the capillary rise region is uniform. From the measured rise $(6 \mu \mathrm{m})$ of the meniscus at the wall we determine the isotropic-nematic surface tension to be $3 \mathrm{nN} / \mathrm{m}$, in quantitative agreement with our theoretical and simulation results.
\end{abstract}

DOI: 10.1103/PhysRevLett.97.087801

Inspired by Langmuir's experiments [1] on platelike clay particles, Onsager put forward a famous entropy argument [2] for the isotropic-nematic (IN) transition of anisometric colloidal particles. The spontaneous orientational ordering of the particles in the nematic phase is attributed to the strongly anisotropic excluded volume pair interactions that favor parallel alignment of the particles and overcome the orientational entropy of the isotropic state. However, the precise particle shape matters, as ordering of thin rods can be quantitatively described at the level of second virial theory, yielding a density jump of $20 \%$ at the IN transition, whereas - to quote Onsager- "we can hardly hope for more than that our result will describe concentrated solutions of (platelike) particles qualitatively rather than quantitatively" [2]. Indeed, computer simulations [3] have shown that for (infinitely thin) hard platelets the density jump is only $8 \%$.

In recent years the entropy-driven IN transition in suspensions of sterically stabilized [4] as well as charge stabilized colloidal platelets [5] has been studied both experimentally and theoretically [6]. To explain that the IN interface is easily deformed under gravity, it was estimated from a scaling relation that the interfacial tension in these systems could be as low as $0.01 \mu \mathrm{N} / \mathrm{m}$ [7]. In this Letter we present a measurement of the IN interfacial tension in suspensions of colloidal platelets and carry out density functional calculations [8] and Monte Carlo (MC) simulations of the free IN interface and wetting at a wall using a microscopic model of platelets with continuous degrees of freedom. For detailed studies of interfacial properties of rectangular platelets with discrete orientations (Zwanzig model) see Refs. $[9,10]$. We find indeed a
PACS numbers: 61.30.Hn, 61.30.Dk, 61.30.Eb, 82.70.Dd

very low IN interfacial tension from the capillary rise measured in experiment, in quantitative agreement with our theoretical and simulation results. Our theory and simulations show complete wetting of the wall by the nematic phase, with its onset occurring only remarkably close to bulk IN coexistence.

We use a model system of hard disks consisting of sterically stabilized colloidal gibbsite $\left[\mathrm{Al}(\mathrm{OH})_{3}\right]$ platelets dispersed in toluene [4], with average diameter $D=$ $240 \mathrm{~nm}$ and thickness $L=18 \mathrm{~nm}$, and a polydispersity of about $20 \%$ in both dimensions. The suspension shows IN coexistence between volume fractions of $23 \%$ and $26 \%$, corresponding to $\rho_{\mathrm{I}} D^{3}=3.8$ and $\rho_{\mathrm{N}} D^{3}=4.3$, where $\rho_{\mathrm{I}}$ $\left(\rho_{\mathrm{N}}\right)$ is the number density in the coexisting I $(\mathrm{N})$ phase. A biphasic sample was contained in a spectrophotometric cell (path length $2.00 \mathrm{~mm}$ ), thoroughly homogenized, and left to phase separate at room temperature. After several days, IN phase separation was complete. The various domains in the nematic phase annealed over several weeks. Then, the sample was investigated with a polarized light microscope and images were recorded with a CCD camera. As shown in Fig. 1(a), when the coexisting $\mathrm{I}$ and $\mathrm{N}$ phases are in contact with a solid vertical wall, the nematic phase exhibits a capillary rise of $h=6 \pm 2 \mu \mathrm{m}$, where $h$ is the height difference of the IN interface in contact with the wall and far away from the wall.

The capillary rise and the shape of the IN interface in the region where the two phases and the wall meet are determined by the interplay between Frank elasticity, surface tension, anchoring strength, and gravity [11]. We have analyzed the Frank elastic energy $F_{E}=\frac{1}{2} \kappa \int d^{3} r[(\nabla \cdot$ $\left.\mathbf{n})^{2}+(\nabla \times \mathbf{n})^{2}\right]$ with the Rapini-Papoular surface free 


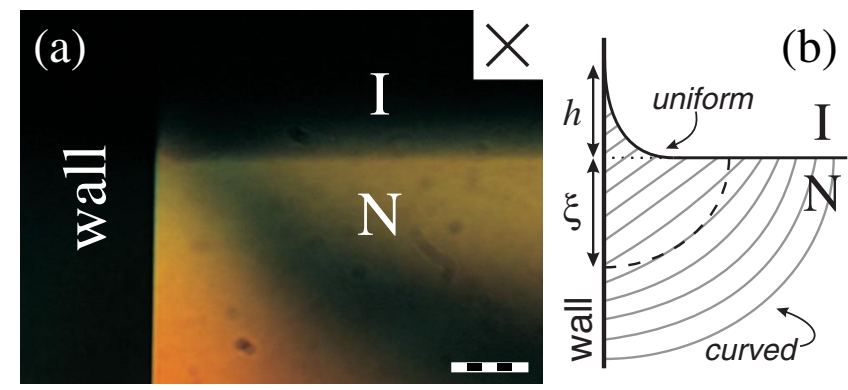

FIG. 1 (color online). (a) Polarized light micrograph of the IN interface. The nematic phase wets the glass wall, yielding a capillary rise of $h=6 \pm 2 \mu \mathrm{m}$. Polarizer orientations are indicated by the cross; the scale bar denotes $50 \mu \mathrm{m}$. (b) Sketch of the IN interface near the capillary-rise region (not to scale). Director field lines are indicated by gray lines. Note that there is a crossover from a uniform director field in the capillary-rise region to a curved director field in the bulk, occurring over a (penetration) length $\xi$.

energy [12] of an $a b$ surface, $F_{a b}=\int d^{2} r\left(\gamma_{a b}+w_{a b} \mid \mathbf{q} \times\right.$ $\left.\mathbf{n}\right|^{2}$ ), with $a, b=\mathrm{I}, \mathrm{N}$, and $\mathrm{W}$ for the isotropic phase, nematic phase, and wall, respectively. Here $\kappa$ is an average Frank elastic constant, $\mathbf{n}(\mathbf{r})$ is the director field at position $\mathbf{r}, \mathbf{q}(\mathbf{r})$ is the interface normal, $\gamma_{a b}$ is the interfacial tension between $a$ and $b$ for $\mathbf{q} \| \mathbf{n}$, and $w_{a b}$ is the $a b$ anchoring strength describing the anisotropy of the interfacial tension. Our simulations and observations show homeotropic anchoring of the nematic phase at both the IN and the WN interface $\left(w_{\mathrm{IN}}, w_{\mathrm{WN}}>0\right)$, hence the platelets tend to lie flat against either interface, as also found for the Zwanzig model [10]. Observing that the dark extinction region lies (roughly) symmetrically between the IN and the WN interface in Fig. 1(a), we infer moreover that $w_{\mathrm{IN}} \simeq w_{\mathrm{WN}} \equiv w$. The equilibrium director field minimizes $F=F_{E}+$ $F_{\mathrm{WI}}+F_{\mathrm{WN}}+F_{\mathrm{IN}}$, and we have studied a wedge with opening angle $\phi$, which for $\phi=\pi / 2$ mimics the experimental geometry of a horizontal IN interface at a vertical wall. In this idealized geometry we find that $\mathbf{n}(\mathbf{r})$ is cylindrically curved for distances from the origin of the wedge $r>\xi \equiv \kappa / w$, such that the IN and WN anchoring is homeotropic, see Fig. 1(b). For smaller distances, $0<r<$ $\xi$, we find a uniform director field with a finite anchoring angle $\phi / 2$ at both interfaces. If the wedge opening angle is $\pi / 2$, the anchoring angle is thus $\pi / 4$. Note that there is no need for a defect because the curved director field escapes into a uniform one [13]. We can now assume that the director field remains homogeneous in the capillary-rise region, provided that $\xi \gg h$.

We have tested this criterion with polarization microscopy of the IN interface in a dispersion contained in a glass capillary with internal cross section $500 \times 500 \mu \mathrm{m}^{2}$, see Fig. 2. The colors reveal the orientation of the director field in the corners and show that its homogeneous parts extend to a distance of about $\xi \simeq 70 \mu \mathrm{m}$. With $\kappa \simeq 7 \times 10^{-14} \mathrm{~N}$ (obtained from experiments on the Frederiks transition
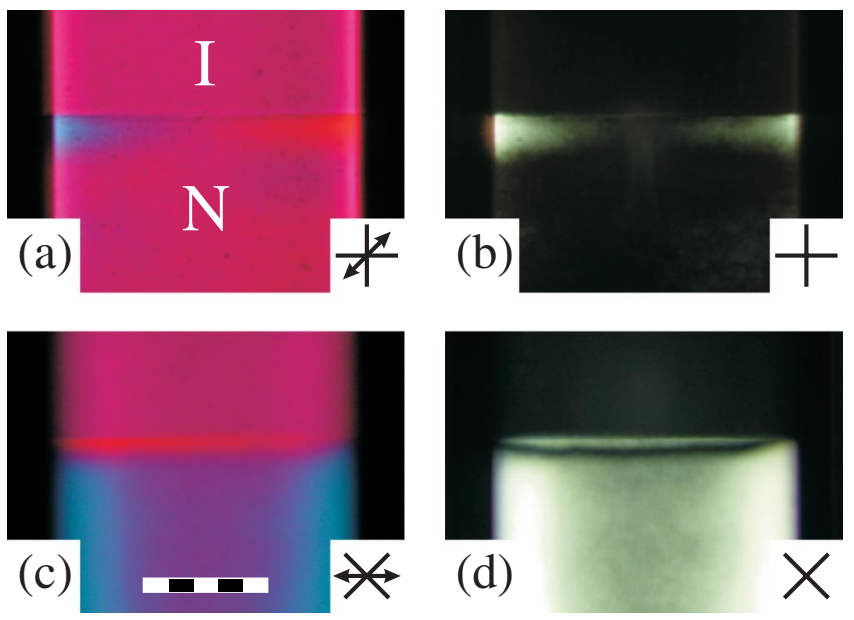

FIG. 2 (color online). Polarized light micrographs of the IN interface, with [(a) and (c)] and without [(b) and (d)] retardation platelet of $530 \mathrm{~nm}$. (a) The blue addition and orange subtraction color in the left and right corners, respectively, indicate perpendicularly aligned director fields. The extent of the birefringent area in (b) indicates the penetration length of the director field of the capillary-rise region, which we determine as $\xi \simeq 70 \mu \mathrm{m}$. Graphs (c) and (d) indicate homeotropic alignment of the nematic phase both at the IN and WN interfaces. Polarizer orientation (crosses) and the slow axis of the retardation platelet (arrows) are indicated; the scale bar denotes $200 \mu \mathrm{m}$ and pertains to all subgraphs.

[14]), we find $w=1 \mathrm{nN} / \mathrm{m}$. Having established that $\xi \gg$ $h$, and hence that the director field in the capillary-rise region is (approximately) homogeneous, we conclude that elastic deformations are of minor importance in the capillary-rise region. The shape of the meniscus is therefore solely governed by the balance between hydrostatic and Laplace pressure and can be obtained analytically using an orientation-averaged surface tension, $\left\langle\gamma_{\mathrm{IN}}\right\rangle$. Assuming complete wetting, we obtain $h \simeq \sqrt{2\left\langle\gamma_{\mathrm{IN}}\right\rangle / g \Delta \rho_{m}}$, where $g$ is the gravitational acceleration and $\Delta \rho_{m}$ is the mass density difference between both phases, similar to the result for isotropic fluids [15]. From the measured height and $\Delta \rho_{m}=20 \mathrm{~kg} \mathrm{~m}^{-3}$ we thus have $\left\langle\gamma_{\mathrm{IN}}\right\rangle=3.5 \mathrm{nN} / \mathrm{m}$. Following Rapini and Papoular, $\left\langle\gamma_{\mathrm{IN}}\right\rangle=\gamma_{\mathrm{IN}}+\frac{1}{2} w$, we obtain the interfacial tension $\gamma_{\mathrm{IN}}=3 \mathrm{nN} / \mathrm{m}=0.04 k_{B} T / D^{2}$, with $T$ temperature and $k_{B}$ the Boltzmann constant.

We next investigate whether the wall is indeed completely wet by the nematic phase by considering the theoretical model of hard circular disks of diameter $D$ in contact with a hard wall. We take the disks to be of vanishing thickness. This does not model the experimental particles very accurately, but may serve as a minimal model to study their wetting properties. Away from the capillary-rise region the one-body distribution, $\rho(z, \vartheta)$, depends only on the perpendicular distance from the interface, $z$, and on the angle $\vartheta$ between the platelet normal and the interface normal. Within density functional theory (DFT) we numerically minimize the grand potential func- 

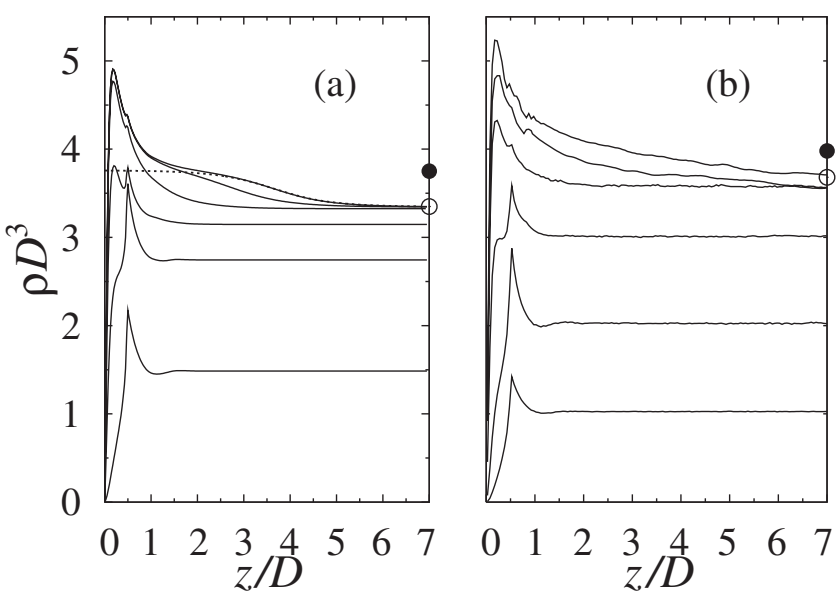

FIG. 3. Scaled density profiles $\rho(z) D^{3}$ in the isotropic phase, obtained from (a) FMT and (b) MC simulations, as a function of the scaled distance from a hard wall, $z / D$, for hard platelets at increasing values of the bulk density (from bottom to top). Bulk isotropic and nematic coexistence densities are indicated by the open and filled circles, respectively. Note the growth of a thick (nematic) wetting layer upon increasing the bulk density. The thickest wetting film in (a) is to be compared with the density profile at a free IN interface (dotted line).

tional, $\tilde{\Omega}[\rho]$, with respect to $\rho(z, \vartheta)$ and hence obtain equilibrium results for $\rho(z, \vartheta)$ and for the grand potential $\Omega=\tilde{\Omega}[\rho]$. We have used either the excess free energy contribution to $\tilde{\Omega}$ from Onsager theory or from fundamental measure theory (FMT) [16]; the latter includes a thirdorder contribution in density and gives a precise account of the bulk IN coexisting densities, $\rho_{\mathrm{I}} D^{3}=3.35, \rho_{\mathrm{N}} D^{3}=$ 3.75 (i.e., a density jump $\sim 10 \%$ ), and nematic order parameter at coexistence, $S=0.53$, as compared to results from simulations: $\rho_{\mathrm{I}} D^{3}=3.68$ and $\rho_{\mathrm{N}} D^{3}=3.98, S=$ $0.45-0.55$ [3], and from Onsager theory: $\rho_{\mathrm{I}} D^{3}=5.33$ and $\rho_{\mathrm{N}} D^{3}=6.79$, and $S=0.78$.

Figure 3(a) displays the orientation-averaged density profile $\rho(z)=\int d \vartheta \sin \vartheta \rho(z, \vartheta)$ at a hard wall as obtained from FMT for increasing bulk densities. While the platelets are depleted from the wall at small bulk densities [8], upon approaching bulk IN coexistence a pronounced nematic peak develops very close to the wall, followed by the growth of a nematic layer into the isotropic bulk. The layer thickness, defined via $d=\int_{0}^{\infty} d z[\rho(z)-\rho(\infty)] /\left(\rho_{N}-\right.$ $\left.\rho_{I}\right)$, grows as $d=-\lambda \ln \left(\left|\mu-\mu_{\text {coex }}\right| / k_{B} T\right)+$ const, where $\mu$ is the chemical potential, and $\mu_{\text {coex }}$ is its value at IN coexistence. The correlation length in the coexisting nematic phase is $\lambda=0.66 D$, which is consistent with $\lambda=$ $0.68 D$ as obtained from the asymptotic decay of the profile of the free IN interface (see Ref. [10] for a similar analysis for the Zwanzig model). The divergence of the film thickness upon approaching bulk IN coexistence indicates complete wetting of the wall by the nematic phase.

In order to test these results we have carried out Monte Carlo simulations using up to $7 \times 10^{4}$ hard plate- lets; large particle numbers are required to stabilize the nematic film against thermal fluctuations, hinting already at a low value of $\gamma_{\mathrm{IN}}$. Density profiles are shown in Fig. 3(b). The similarity with the theoretical profiles is striking, confirming the growth of a pronounced nematic wetting layer. Its decay into the isotropic bulk, $z / D \rightarrow \infty$, is somewhat more diffuse than that found in DFT, which we attribute to the presence of additional (capillary wave) fluctuations in the simulations.

The interfacial tension is defined as $\gamma=(\Omega+p V) / A$, where $\Omega$ is the grand potential of the inhomogeneous system, $p$ the bulk pressure, $V$ the volume, and $A$ the interface area. In simulations we use thermodynamic integration to determine the free energy difference of a bulk system and a system with a wall. We approximate the hard wall by a finite barrier, such that a zero barrier corresponds to a bulk system and an infinite barrier to a system with a hard wall [17]. This yields the wall tensions $\gamma_{\mathrm{WI}}$ and $\gamma_{\mathrm{WN}}$ and we obtain $\gamma_{\mathrm{IN}}$ via Young's equation, $\gamma_{\mathrm{WN}}-\gamma_{\mathrm{WI}}=$ $\gamma_{\mathrm{IN}} \cos \theta$, where the contact angle $\theta$ of the free IN interface and the wall vanishes for complete wetting, $\theta=0$. Thus we find $\gamma_{\mathrm{IN}} \simeq 0.06 k_{B} T / D^{2}$ from simulations (for $\theta=0$ ). From FMT, we obtain $\gamma_{\mathrm{IN}}=0.03 k_{B} T / D^{2}$. Both values are in good agreement with the experimental value of $0.04 k_{B} T / D^{2}$, in particular, given that the gibbsite platelets are polydisperse in size. From Onsager theory we obtain $\gamma_{\mathrm{IN}}=0.20 k_{B} T / D^{2}$, overestimating the above results, which is not surprising in view of its shortcomings for the bulk transition densities.

We find the initial increase of the wall tension $\gamma$ with $\rho=\rho(\infty)$ in the isotropic phase to be very well described by the result obtained from applying scaled-particle theory [18], $\gamma / k_{B} T=\pi D \rho / 8+\pi D^{4} \rho^{2} / 32$, see Fig. 4(a). As IN coexistence is approached, $\gamma$ exhibits a maximum and decreases towards its value at the isotropic coexistence density, $\gamma_{\mathrm{WI}}$. This nonmonotonic behavior is accompanied by the growth of the nematic wetting layer leading to a reduction of the interfacial tension. In the nematic phase $\gamma$ decreases weakly as a function of $\rho$. Although the Onsager functional overestimates the behavior in the coexistence region significantly, it leads to qualitatively similar behavior.

In order to simulate the free IN interface directly, we have used penetrable cut spheres with diameter-tothickness ratio $D / L=30$; overlaps between platelets are penalized with an energy cost $\epsilon=1 k_{B} T$, irrespective of the particle orientation. This interaction leads to a higher IN transition density and a larger density jump. Following Ref. [19], the probability distribution of the particle density, $P(\rho)$, was obtained; it becomes bimodal at coexistence, see Fig. 4(b). The locations of the peaks yield the densities of the coexisting phases, the peak height in $\ln [P(\rho)]$ is used to measure $\gamma_{\mathrm{IN}}$. Using two different system sizes, we have extrapolated to the thermodynamic limit and find $\gamma_{\mathrm{IN}} \approx 0.2 k_{B} T / D^{2}$, which, as expected, exceeds the result for hard platelets. 

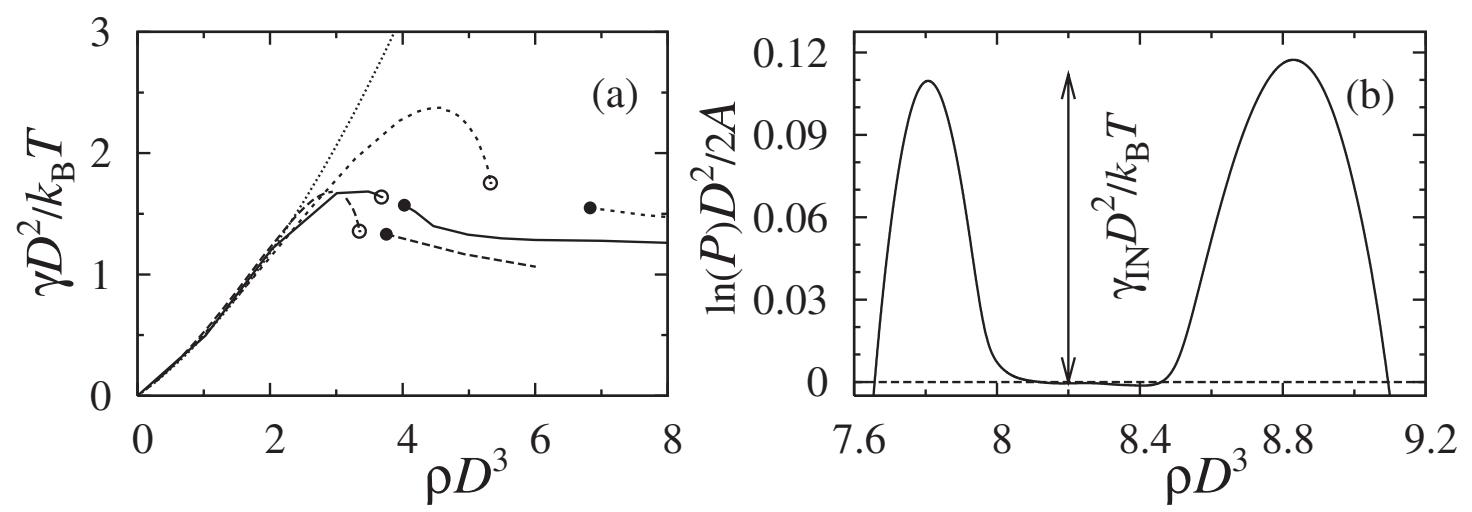

FIG. 4. (a) The wall interfacial tension $\gamma D^{2} / k_{B} T$ versus scaled density $\rho D^{3}$, as obtained from FMT (long dashed line), Onsager theory (short dashed line), scaled-particle theory (dotted line), and simulations (full line). Also shown are the wall-isotropic tension, $\gamma_{\mathrm{WI}}$ (open circles), and wall-nematic tension, $\gamma_{\mathrm{WN}}$ (filled circles), at bulk IN coexistence, whose difference yields $\gamma_{\mathrm{IN}}$. (b) Scaled logarithm of the density distribution, $\ln [P(\rho)] D^{2} / 2 A$, versus $\rho D^{3}$ for penetrable platelets at bulk coexistence, with $A=49 D^{2}$ the lateral area of the simulation box. The peak height reflects $\gamma_{\mathrm{IN}}$ (arrow) [19].

In conclusion, we have performed a study of the isotropic-nematic interface in dispersions of hard disks using experiment, theory, and computer simulations. The interfacial tension of the IN interface of hard disks was measured to be $\gamma_{\mathrm{IN}}=3 \mathrm{nN} / \mathrm{m}$. The good agreement of this low value with our theoretical and simulation results, where we have used infinitely thin circular platelets and have not incorporated polydispersity, may be somewhat fortuitous. These features are known to influence the bulk phase behavior significantly and therefore one might expect that they also influence interfacial properties $[7,9,10]$. The low value of $\gamma_{\mathrm{IN}}$ and complete wetting of a hard wall are in agreement with studies of the Zwanzig model $[9,10]$ (incorporating model polydispersity and finite thickness of the platelets). Our work opens up avenues for further studies of interesting interfacial phenomena like tactoid coalescence and capillary waves at anisotropic interfaces [20].

We thank R. Blaak for helpful comments and D. Aarts for experimental assistance. We acknowledge support of the Nederlandse Organisatie voor Wetenschappelijk Onderzoek (NWO), the MWFZ Forschungsfonds, the Deutsche Forschungsgemeinschaft (SFB-TR6/D3 and D5, and the Emmy Noether program), as well as the Forschungszentrum Jülich for allocation of CPU time. This work is part of the research program of the Stichting voor Fundamenteel Onderzoek der Materie (FOM), financially supported by NWO.

*Present address: Laboratoire de Chimie de la Matière Condensée, Université Pierre et Marie Curie (Paris VI), 4 place Jussieu, Case courrier 174, 75252 Paris Cedex 05, France.

Electronic address: vdbeek@ccr.jussieu.fr
[1] I. Langmuir, J. Chem. Phys. 6, 873 (1938).

[2] L. Onsager, Ann. N.Y. Acad. Sci. 51, 627 (1949).

[3] R. Eppenga and D. Frenkel, Mol. Phys. 52, 1303 (1984); J. A. C. Veerman and D. Frenkel, Phys. Rev. A 45, 5632 (1992); M. A. Bates and D. Frenkel, J. Chem. Phys. 110, 6553 (1999).

[4] F. M. van der Kooij and H. N. W. Lekkerkerker, J. Phys. Chem. B 102, 7829 (1998); F. M. van der Kooij et al., Nature (London) 406, 868 (2000).

[5] D. van der Beek and H. N. W. Lekkerkerker, Europhys. Lett. 61, 702 (2003); Langmuir 20, 8582 (2004); S. Liu et al., Chem. Mater. 15, 3240 (2003).

[6] L. Harnau et al., Europhys. Lett. 53, 729 (2001).

[7] F. M. van der Kooij et al., J. Phys. Chem. B 105, 1696 (2001).

[8] L. Harnau and S. Dietrich, Phys. Rev. E 65, 021505 (2002).

[9] L. Harnau and S. Dietrich, Phys. Rev. E 66, 051702 (2002); M. Bier, L. Harnau, and S. Dietrich, J. Chem. Phys. 123, 114906 (2005).

[10] M. Bier, L. Harnau, and S. Dietrich, Phys. Rev. E 69, 021506 (2004).

[11] A. D. Rey, Langmuir 19, 3677 (2003).

[12] A. Rapini and M. Papoular, J. Phys. (Paris), Colloq. 30, C4-54 (1969).

[13] A. Poniewierski, Liq. Cryst. 27, 1369 (2000).

[14] D. van der Beek et al. (unpublished).

[15] See, e.g., J.S. Rowlinson and B. Widom, Molecular Theory of Capillarity (Clarendon, Oxford, 1982), Chap. 1.

[16] A. Esztermann et al., Phys. Rev. E 73, 011409 (2006).

[17] A. Fortini et al., Phys. Rev. E 71, 051403 (2005).

[18] See, e.g., S. M. Oversteegen and R. Roth, J. Chem. Phys. 122, 214502 (2005).

[19] R. L. C. Vink et al., J. Chem. Phys. 123, 074901 (2005); K. Binder, Phys. Rev. A 25, 1699 (1982).

[20] N. Akino, F. Schmid, and M. P. Allen, Phys. Rev. E 63, 041706 (2001). 